\title{
roAp stars: surface lithium abundance distribution and magnetic field configuration
}

\author{
N. Polosukhina ${ }^{1}$, D. Shulyak ${ }^{2}$, A. Shavrina ${ }^{3}$, D. Lyashko ${ }^{4}$, \\ N. A. Drake ${ }^{5,6}$, Yu. Glagolevski ${ }^{7}$, D. Kudryavtsev ${ }^{7}$ and M. Smirnova ${ }^{1}$ \\ ${ }^{1}$ Crimean Astrophysical Observatory, Nauchnyi, Ukraine, email: polo@crao.crimea.ua \\ ${ }^{2}$ Georg-August University, Göttingen, Germany, email: denis.shulyak@gmail.com \\ ${ }^{3}$ Main Astronomical Observatory of NAS of Ukraine, Kyiv, Ukraine, email: \\ shavrina@mao.kiev.ua \\ ${ }^{4}$ Taurida National V. I. Vernadsky University, Simferopol, Crimean Autonomous Republic, \\ Ukraine, email: dlyashko@gmail.com \\ ${ }^{5}$ Sobolev Astronomical Institute, St. Petersburg State University, St. Petersburg, Russia \\ ${ }^{6}$ Observatório Nacional/MCTI, Rio de Janeiro, Brazil, email: drake@on.br \\ ${ }^{7}$ Special Astrophysical Observatory of RAS, Nizhnii Arhyz, Russia, email: dkudr@sao.ru
}

\begin{abstract}
High-resolution spectra obtained with the 6m BTA telescope, Russia, and with HARPS and VLT/UVES telescopes at ESO, Chile, were used for Doppler Imaging analysis of two roAp stars, HD 12098 and HD 60435, showing strong and variable Li resonance line in their spectra. We found that Li has highly inhomogeneous distribution on the surfaces of these stars. We compared our results with previously obtained Doppler Imaging mapping of two CP2 stars, HD 83368 and HD 3980, and discuss the correlation between the position of the high Li-abundance spots and magnetic field.
\end{abstract}

Keywords. stars: abundances - stars: chemically peculiar - stars: magnetic field - stars: individual: HD 3980, HD 12098, HD 60435, HD 83368

\section{Introduction}

Spectral observations of a number of magnetic chemically peculiar stars allowed us to discover several Ap stars with abnormally high lithium abundance. However, the behaviour of the Li I $6708 \AA$ line in these stars is still puzzling (Polosukhina et al. 1999, 2004, 2012).

In this paper we present the first results of the Doppler Imaging of two rapidly oscillating (roAp) cool magnetic chemically peculiar stars, HD 12098 and HD 60435, showing a remarkable rotational modulation of the Li I line. The high-resolution spectra of the northern star HD 12098 were obtained during 2007 - 2013 with the 6m BTA telescope of SAO, Russia, whereas the HARPS spectra of HD 60435 were downloaded from the ESO Science Archive Facility under request number drake.62954.

\section{First results and conclusions}

For the investigation of the surface distribution of lithium on the surfaces of HD 12098 and HD 60435, we used the Doppler Imaging (DI) code INVERS12 (Piskunov \& Rice 1993; Kochukhov et al. 2004). We modelled the resonance Li I doublet at $\lambda 6708 \AA$ taking into account its variable blending with the nearby line of Pr III $6706.7 \AA$.

Our earlier DI analysis of two other roAp stars, HD 83368 (Kochukhov et al. 2004) and HD 3980 (Nesvacil et al. 2012), showed that Li is strongly concentrated in the areas of the magnetic poles and depleted in the regions around the magnetic equator. 


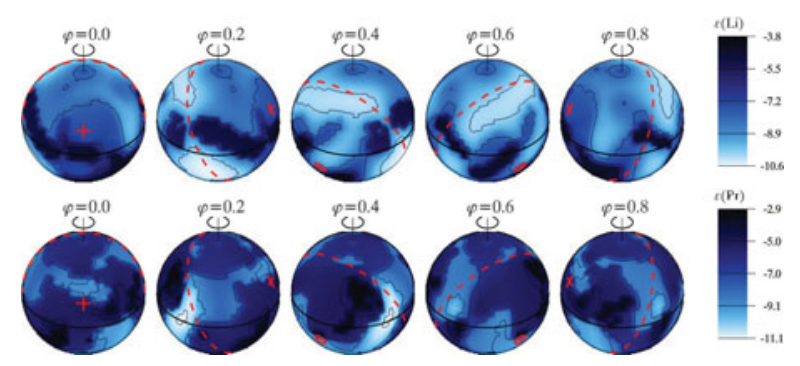

Figure 1. Distribution of $\mathrm{Li}$ and $\mathrm{Pr}$ on the surface of HD 12098. The star is shown at five rotation phases. The abundance scales are in $\log N(\mathrm{el}) / N(\mathrm{H})$ (darker areas correspond to higher element abundance). The dashed line, plus sign and small circle correspond to the magnetic equator, positive and negative poles respectively. The following stellar parameters were used: $T_{\text {eff }}=7800 \mathrm{~K}, \log g=4.3, v \sin i=10 \pm 2 \mathrm{~km} \mathrm{~s}^{-1}, B_{\mathrm{p}}=6.5 \mathrm{kG}, i \cong 55^{\circ}, \beta=65^{\circ}$ (Shavrina et al. 2008), $P_{\text {rot }}=5.460^{\mathrm{d}} \pm 0.001$ (Ryabchikova et al. 2005 ).

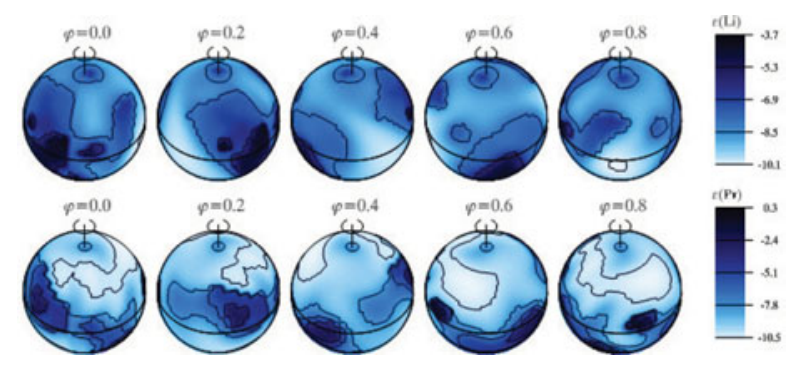

Figure 2. The same as in Fig. 1, but for the star HD 60435. The following stellar parameters were used: $T_{\text {eff }}=8250 \mathrm{~K}, \log g=4.5, v \sin i=11 \pm 2 \mathrm{~km} \mathrm{~s}^{-1}, H_{\mathrm{s}}=3 \pm 1 \mathrm{kG}, i \cong 47^{\circ}\left(133^{\circ}\right)$ (Shavrina et al. 2001), $P_{\text {rot }}=7.6793^{\mathrm{d}} \pm 0.0006$ (Kurtz et al. 1990 )

Preliminary DI of HD 12098 showed that Li has highly inhomogeneous distribution on the surface of this star. However, no such correlation has been found between the magnetic field structure and the Li abundance. For the star HD 60435 the picture is uncertain because of the lack of magnetic field model for this star.

Analysis of the Li I line behaviour in magnetic CP stars can give new challenges to the theoretical calculations of diffusion in the presence of strong magnetic fields. New observations of HD 12098 permitting to achieve the better phase coverage are still needed.

Acknowledgements. N. A. D. acknowledges support of the PCI/MCTI, Brazil, under the Project 302350/2013-6.

\section{References}

Kochukhov, O., Drake, N. A., Piskunov, N., \& de la Reza, R. 2004, A\&\&A, 424, 935

Kurtz, D. W., van Wyk, F., Marang, F. 1990 MNRAS, 243, 289

Nesvacil, N., Lüftinger, T., Shulyak, D. et al. 2012, A\&\&A, 537, A151

Piskunov, N. E. \& Rice, J. B. 1993, PASP, 105, 1415

Polosukhina, N., Kurtz, D., Hack, M. et al. 1999, A\&A, 351, 283

Polosukhina, N., Shavrina, A., Drake, N. A. et al. 2004, A-Star Puzzle, Proc. IAU Symp. 224 , Cambridge University Press, 2004, 665

Polosukhina, N., Shavrina, A., Drake, N. A., \& Smirnova, M. 2012, MSAIS, 22, 129

Ryabchikova, T., Wade, G. A., Aurière, M. et al. 2005, A\&A, 429, 55

Shavrina, A. V., Polosukhina, N. S., Zverko, J. et al. 2001, A\& A, 372, 571

Shavrina, A. V., Polosukhina, N. S., Drake, N. A., \& Kudryavtsev, D. O. 2008, Astrophysics, 51,517 